\title{
Epithelial-myoepithelial carcinoma of the nasopharynx: A case report and review of the literature
}

\author{
SUL-HEE KIM ${ }^{1}$, SUNG-EN PARK ${ }^{1}$, HYUNG-GI BAE ${ }^{1}$, DOO-HAN SONG ${ }^{1}$, HYUN-HO OH $^{1}$, \\ KYOUNG-RAI CHO ${ }^{2}$, HYUN-JUNG KIM ${ }^{3}$ and BYEONG SEOK SOHN ${ }^{1}$ \\ Departments of ${ }^{1}$ Internal Medicine, ${ }^{2}$ Otolaryngology and ${ }^{3}$ Pathology, Sanggye Paik Hospital, \\ Inje University College of Medicine, Seoul 139-707, Republic of Korea
}

Received September 3, 2014; Accepted May 15, 2015

DOI: $10.3892 / \mathrm{ol} .2015 .3314$

\begin{abstract}
An 80-year-old female with complaints of right-sided nose blockage and occasional epistaxis for several months was referred to Sanggye Paik Hospital (Seoul, Korea). Nasal examination revealed a hemorrhagic mass protruding towards the right posterior nasal cavity, for which the patient was subjected to excisional biopsy. The pathological diagnosis was epithelial-myoepithelial carcinoma, as immunohistochemical analysis revealed that the inner cells stained positive for cytokeratin 7, an epithelial cell marker, whereas the outer cells stained positive for smooth muscle actin and P63, consistent with a myoepithelial phenotype. The patient received concurrent chemoradiation therapy followed by systemic chemotherapy. Following the achievement of a partial response, the patient's treatment course was uneventful. Follow-up at 24 months revealed a stable status at partial response, with no subsequent progression.
\end{abstract}

\section{Introduction}

Epithelial-myoepithelial carcinoma (EMCa) is a malignant biphasic salivary-type tumor, which is typically composed of large, clear myoepithelial cells surrounding variable proportions of epithelial-lined ducts that resemble intercalated ducts $(1,2)$. EMCa predominantly arises in the parotid gland, accounting for $\sim 1 \%$ of all salivary gland tumors (2-4). Immunohistochemical studies are useful in the differentiation of EMCa from other similar tumors, highlighting their characteristic biphasic epithelial-myoepithelial phenotype (1).

However, several cases of EMCa have been reported in the mucoserous glands of the upper and lower aerodigestive tract $(1,3)$, including the paranasal sinuses (2), nasopharynx (3),

Correspondence to: Dr Byeong Seok Sohn, Department of Internal Medicine, Sanggye Paik Hospital, Inje University College of Medicine, 1342 Dongilro, Seoul 139-707, Republic of Korea E-mail:imbs@paik.ac.kr

Key words: epithelial-myoepithelial carcinoma, chemotherapy, immunohistochemistry, chemoradiation therapy nasal cavity (2), hypopharynx (4), larynx (4), trachea $(2,4)$, bronchus (2) and lungs (5). Additionally, the development of EMCa at unusual sites, for example the breast (2) and lacrimal glands $(2,4)$, has been reported.

The present study reports a rare case of nasopharyngeal EMCa in an 80-year-old woman treated with concurrent chemoradiation therapy, followed by systemic chemotherapy.

\section{Case report}

An 80-year-old woman with complaints of right-sided nose blockage and occasional epistaxis for several months was referred to Sanggye Paik Hospital (Seoul, Korea). The patient had a history of diabetes mellitus and hypertension for several years, but no notable history of rhinologic problems other than a Caldwell-Luc operation to treat chronic maxillary sinusitis 20 years previously. On nasal speculum examination, a hemorrhagic mass protruding towards the right posterior nasal cavity was observed. There was no cervical lymphadenopathy detected on physical examination. The results of laboratory examination including complete blood count, electrolytes, and urine analysis were normal. Chest radiographs also revealed no significant findings. Computed tomography (CT) of the neck revealed a heterogeneously enhancing soft tissue mass in the right nasopharynx (Fig. 1), and two enhanced ovoid lymph nodes of $<1 \mathrm{~cm}$ in the retropharyngeal space, with unknown clinical significance. There was no hot-uptake lesion except for the right nasopharyngeal mass in positron emission tomography-CT (PET-CT). Excisional biopsy was performed under general anesthesia.

Histopathological findings. Nasal endoscopic examination revealed an easily bleeding, friable polypoid mass in the nasopharynx, which completely obstructed the right choana (Fig. 2). The histopathological diagnosis of the specimen from excisional biopsy was EMCa. Grossly, multiple fragments of the cellular, lobulated mass were separated by sclerotic septae, against a background indicative of recent hemorrhage, and the tissue was lined by respiratory epithelium. The majority of the mass was composed of uniform clear cells and partially of glandular components. Hematoxylin and eosin staining revealed that a major portion of the tumor was composed of solid-growing, oval, clear cells, nested within periodic 


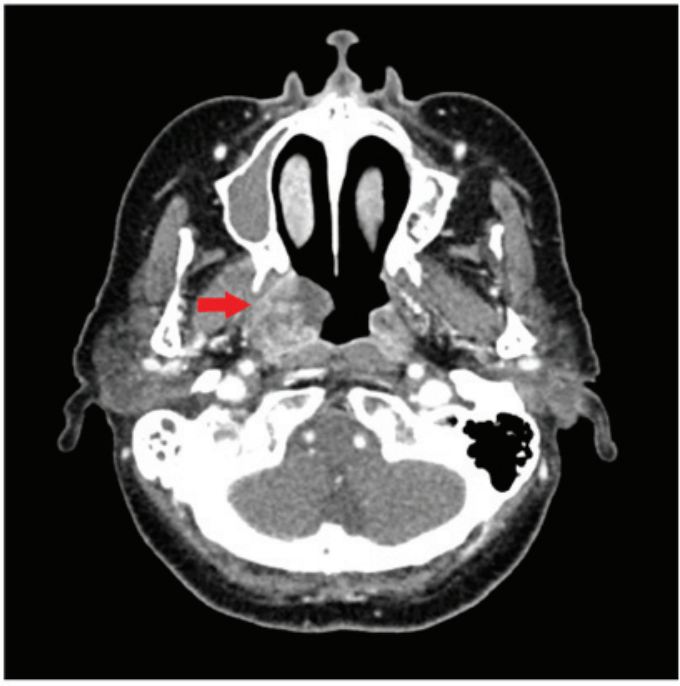

Figure 1. Computed tomography of the neck. A heterogeneously enhancing mass with a lobulated contour was detected in the right nasopharynx

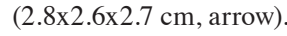

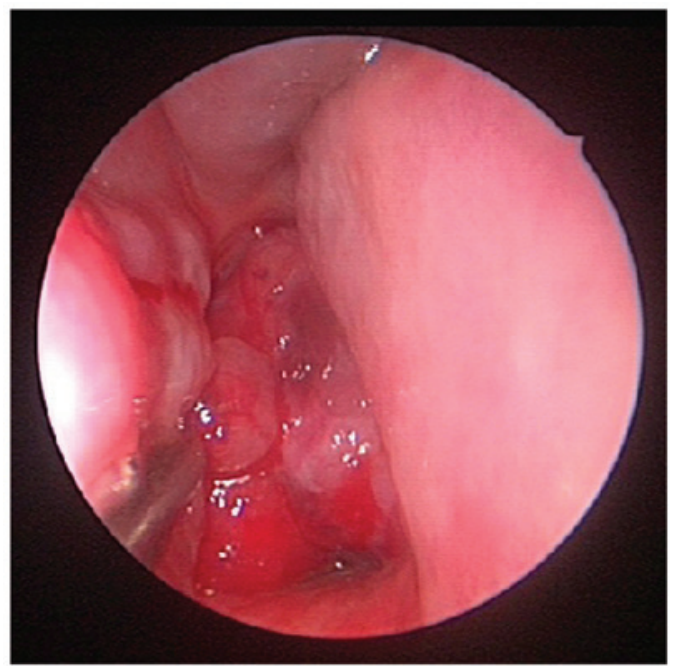

Figure 2. Nasal endoscopic examination revealed an easily bleeding, friable polypoid mass protruding into the right posterior nasal cavity, with complete obstruction of the right choana.

acid-Schiff (PAS) stain-positive basement membrane-like material (Fig. 3A). The minor section demonstrated bi-layered duct-like structures with an inner layer of a single row of cuboidal cells and an outer layer of a single or multiple layers of oval cells, a hallmark histological feature of EMCa (Fig. 3B). Immunohistochemical staining revealed that the inner cells stained positive for cytokeratin 7, an epithelial cell marker, whereas the outer oval cells were positive for P63 (nuclear expression pattern; Fig. 3C) and smooth muscle actin (cytoplasmic expression pattern; Fig. 3D), consistent with a myoepithelial phenotype, confirming the diagnosis of EMCa. There was no nuclear atypia or increased mitotic activity. However, infarct-type necrosis was observed in the excisional biopsy specimen.

Treatment and outcomes. The patient received concurrent chemoradiation therapy followed by systemic chemotherapy.
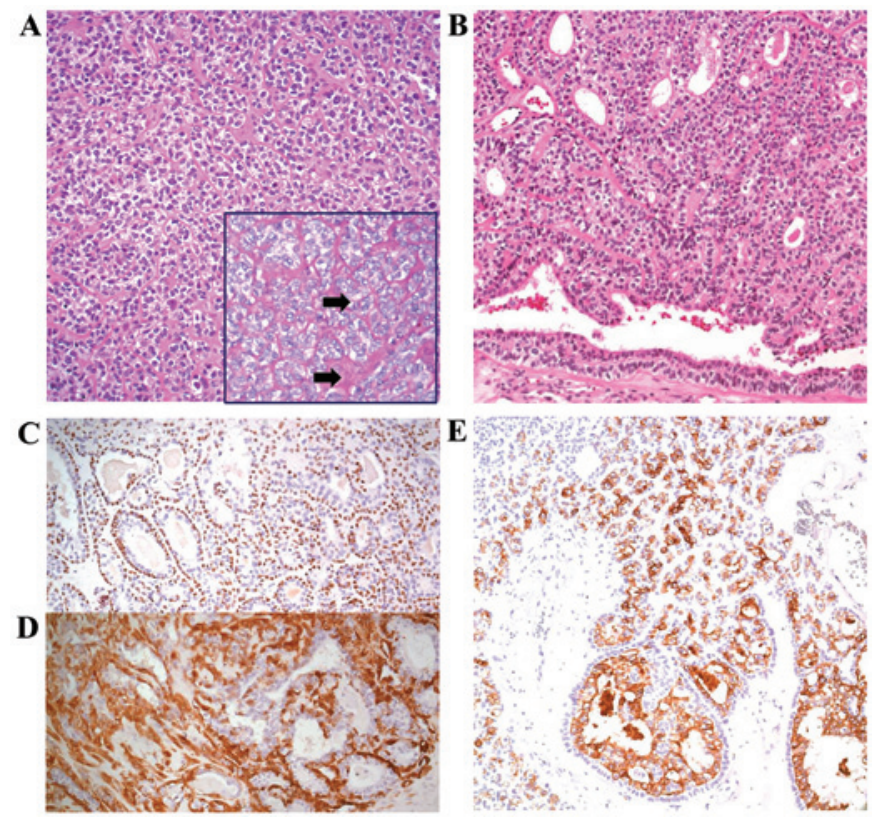

Figure 3. Pathological findings. (A) Hematoxylin and eosin staining identified basement membrane-like material (arrows, inset) and (B) bi-layered duct-like structures. (C) Immunohistochemical staining identified P63 (nuclear expression pattern), (D) smooth muscle actin (cytoplasmic expression pattern) and (E) cytokeratin 7 (arrows, inset, PAS; magnification, x200).

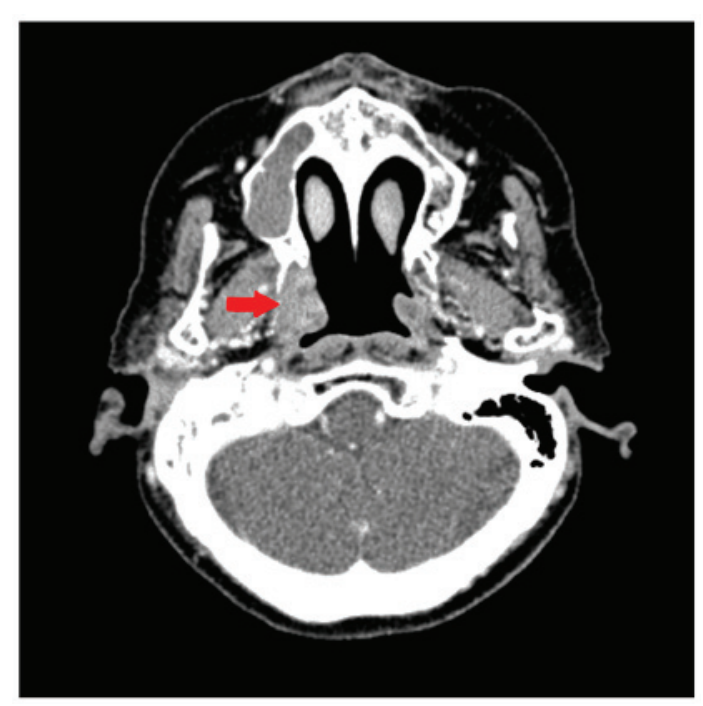

Figure 4. Computed tomography scan of the neck at follow-up 24 months post-treatment. No significant change was detected in the size of the tumor following the achievement of a partial response (arrow).

Volumetric modulated arc therapy was administered with a total dose of $66.6 \mathrm{~Gy}$ in 37 fractions (180 cGy/fraction, 5 fractions/week). During radiation therapy, $40 \mathrm{mg} / \mathrm{m}^{2}$ cisplatin was administrated weekly, for up to 4 cycles. The patient achieved a partial response, as observed in a neck CT performed 8 weeks subsequent to the completion of concurrent chemoradiation therapy (Fig. 4). Subsequently, the patient was treated with a systemic chemotherapy regimen that included cisplatin (45 mg/m $\mathrm{m}^{2}$, day 1$)$ and 5 -fluorouracil $\left(750 \mathrm{mg} / \mathrm{m}^{2}\right.$, days $\left.1-4\right)$ at 4 -week intervals. Following the completion of 2 cycles of systemic chemotherapy, no further tumor shrinkage was observed. Due to the patient's advanced age, poor performance 
status and intolerance to chemotherapy toxicity, systemic chemotherapy was discontinued following completion of 2 cycles. Thereafter, the post-treatment course was uneventful. Follow-up at 24 months revealed a stable status with no progression following the achievement of a partial response.

\section{Discussion}

EMCa, first described by Donath et al (6) in 1972, is a rare and unique tumor, accounting for $\sim 1 \%$ of all epithelial salivary gland neoplasms $(4,7)$. The mean age at presentation is 60.9 years, with a female predominance (1.5:1) (1). According to the World Health Organization classification of salivary gland tumors, EMCa is defined as a distinctive subtype of malignant epithelial tumors, consisting of varying proportions of two cell types (8).

The diagnosis of EMCa is typically based on conventional light microscopy findings and confirmed by immunohistochemistry (8). The tumor is composed of nests that are partitioned by reticulin fibers. Each nest is comprised of duct-forming cells bordering a lumen, and cells surrounding the duct-forming cells. Dark cells with few organelles form the inner layer of the tubules (similar to those of the intercalated ducts), and clear cells rich in organelles (myofilaments, endoplasmic reticulum, pinocytosis vesicles, as well as glycogen and lipofuscin granules) and glycogen form the outer layer with myoepithelial differentiation (6). Immunohistochemical studies may be useful in facilitating the differentiation of EMCa from similar tumors via identification of the characteristic bi-layered epithelial-myoepithelial phenotype (1). On immunohistochemical staining, the epithelial cells are positive for epithelial markers, including cytokeratins and epithelial membrane antigen, but negative for myoepithelial cell markers, for example $\alpha$-smooth muscle actin, P63 and vimentin, thus indicating their epithelial nature (2). By contrast, the cells surrounding these duct-forming cells exhibit antagonistic characteristics; they are positive for myoepithelial cell markers but negative for epithelial cell markers, demonstrating their myoepithelial nature $(2,9)$.

EMCa typically exhibits low-grade malignancy and is associated with a favorable prognosis. However, aggressive histological features, local recurrence, regional metastasis and even mortalities are not uncommon $(1,2,4,8)$. The most significant univariate factors in the prediction of EMCa recurrence are margin status, angiolymphatic invasion, tumor necrosis and myoepithelial anaplasia (1). The EMCa recurrence rate is $36.3 \%(1,2)$. Regional lymph node metastasis and distant metastasis to the lungs, skin, kidney, bone and brain have been reported in $\sim 5 \%$ of cases $(2,3,10)$. The 5- and 10-year disease-specific survival rates are 93.5 and $81.8 \%$, respectively (1).

There is currently no consensus regarding the optimal treatment for this salivary gland neoplasm, largely due to its rarity (10). Wide surgical excision with a clear margin has been recommended $(2-4,7,10,11)$. As with other malignant head and neck tumors, EMCa tumors have a propensity for infiltration and local recurrence. Therefore, adjuvant radiotherapy has been recommended, particularly for neoplasms with a diameter of $>4 \mathrm{~cm}$, to aid the prevention of local recurrence $(4,10,11)$. The effect of chemotherapy in the treatment of EMCa remains unclear $(11,12)$.

In the present case, due to the anatomical location, the use of radiation therapy was suggested. In addition, the tumor specimen revealed infarct-type necrosis, a risk factor for recurrence; hence concurrent chemotherapy was added to the radiation therapy treatment plan. Cisplatin, 5-fluorouracil, paclitaxel and cyclophosphamide are frequently used in the treatment of other histological subtypes of salivary gland neoplasms; of these, cisplatin was selected for concurrent chemoradiation therapy to treat the present patient $(11,13)$. Thereafter, subsequent systemic chemotherapy with cisplatin and 5-fluorouracil at 4-week intervals was planned. Although there are few studies on the treatment of EMCa with a combination of radiation therapy and chemotherapy, two reports of EMCas originating from the tongue base demonstrated a positive treatment response (complete response) with sequential chemoradiation and radiation therapy, respectively $(13,14)$. In addition, in one case report of an EMCa of the submandibular gland with lung metastasis, treatment with cisplatin combined with 5-fluorouracil, and followed by paclitaxel resulted in disease stabilization and relief of pulmonary symptoms (11). Following treatment, the partial response has been maintained for 24 months. However, we cannot conclusively determine whether the long-term response was due to the low-grade malignant potency of the tumor, the effect of concurrent chemoradiation therapy or systemic chemotherapy.

To the best of our knowledge, except for a case report of EMCa in the nasopharynx, which was treated by surgical resection (3), the present study reports the only case of EMCa in the nasopharynx treated with concurrent chemoradiation therapy followed by systemic chemotherapy, in which a partial response was achieved. Although the optimal treatment strategy for EMCa remains poorly defined due to its rarity, concurrent chemoradiation therapy followed by systemic chemotherapy may offer an alternative or mainstay of treatment for EMCa as squamous cell carcinoma or a variant of the nasopharynx.

\section{References}

1. Seethala RR, Barnes EL and Hunt JL: Epithelial-myoepithelial carcinoma: A review of the clinicopathologic spectrum and immunophenotypic characteristics in 61 tumors of the salivary glands and upper aerodigestive tract. Am J Surg Pathol 31: 44-57, 2007.

2. Yamanegi K, Uwa N, Hirokawa M, Ohyama H, Hata M, Yamada N, Ogino K, Toh K, Terada T, Tanaka A, et al: Epithelial-myoepithelial carcinoma arising in the nasal cavity. Auris Nasus Larynx 35: 408-413, 2008.

3. Imate Y, Yamashita H, Endo S, Okami K, Kamada T, Takahashi $\mathrm{M}$ and Kawano $\mathrm{H}$ : Epithelial-myoepithelial carcinoma of the nasopharynx. ORL J Otorhinolaryngol Relat Spec 62: 282-285, 2000.

4. Park JO, Jung CK, Sun DI and Kim MS: An unusual presentation of aggressive epithelial-myoepithelial carcinoma of the nasal cavity with high-grade histology. J Laryngol Otol 125: 1286-1289, 2011

5. Cho SH, Park SD, Ko TY, Lee HY and Kim JI: Primary epithelial myoepithelial lung carcinoma. Korean J Thorac Cardiovasc Surg 47: 59-62, 2014.

6. Donath K, Seifert G and Schmitz R: Diagnosis and ultrastructure of the tubular carcinoma of salivary gland ducts. Epithelial-myoepithelial carcinoma of the intercalated ducts. Virchows Arch A Pathol Pathol Anat 356: 16-31, 1972 (In German). 
7. Pradhan SA, Khannan R, Hazarika B and Desai M: Sinonasal epithelial-myoepithelial carcinoma - a rare entity. Indian J Otolaryngol Head Neck Surg 59: 168-170, 2007.

8. Barnes L, Eveson, JW, Reichart P and Sidransky D (eds): Pathology and Genetics of Head and Neck Tumours. In: World Health Organization Classification of Tumours. Vol 9. IARC Press, Lyon, 2005.

9. Lavanya N, Joshua E and Ranganathan K: Epithelial myoepithelial carcinoma. J Oral Maxillofac Pathol 16: 473-474, 2012.

10. Lee HM, Kim AR and Lee SH: Epithelial-myoepithelial carcinoma of the nasal cavity. Eur Arch Otorhinolaryngol 257: 376-378, 2000

11. Pierard S, Gregoire V, Weynand B and Machiels JP: Epithelial-myoepithelial carcinoma of the submandibular gland with symptomatic lung metastases treated with chemotherapy. Eur Arch Otorhinolaryngol 263: 1158-1160, 2006.
12. Kuran G, Sagit M, Akin I, Hucumenoglu S, Ocal BG and Celik SY: Bilateral epithelial-myoepithelial carcinoma: An extraordinary tumor of the paranasal sinuses. Skull Base 18: $145-150,2008$

13. Puri T, Singh K, Sharma DN and Khurana $\mathrm{N}$ Epithelial-myoepithelial carcinoma of the base of tongue: Pathology and management. Indian J Cancer 41: 138-140, 2004.

14. Peters P, Repanos C, Earnshaw J, Stark P, Burmeister B, McGuire L, Jeavons $S$ and Coman A M WB: Epithelial-myoepithelial carcinoma of the tongue base: A case for the case-report and review of the literature. Head Neck Oncol 2: 4, 2010 